\title{
Optical Band Gap Estimation of ZnO Nanorods
}

\author{
Angélica Sáenz-Trevizo ${ }^{a *}$, Patricia Amézaga-Madrid ${ }^{a}$,Pedro Pizá-Ruiz ${ }^{a}$,Wilber Antúnez-Flores ${ }^{a}$,
}

\author{
Mario Miki-Yoshida ${ }^{a}$ \\ ${ }^{a}$ Centro de Investigación en Materiales Avanzados S.C., Chihuahua, Chihuahua, CP 31136, México
}

Received: October 9, 2015; Revised: April 14, 2016; Accepted: July 8, 2016

The optical band gap energy of $\mathrm{ZnO}$ nanorods was estimated by different methods found in literature. These nanorods were deposited onto $\mathrm{TiO}_{2}$ covered borosilicate glass substrates by aerosol assisted chemical vapor deposition. Characterization techniques were employed to reveal the crystalline structure, surface morphology and optical properties of the sample. The analysis proved that hexagonal cross-section $\mathrm{ZnO}$ nanorods in the wurtzite phase were grown onto a $\mathrm{TiO}_{2}$ buffer thin film of anatase. Variations in the components of transmittance and reflectance were associated with the development of a rough surface. Such fluctuations were correlated with the equations employed to estimate the optical band gap. The different methods indicated band gap values of 3.17 to $3.24 \mathrm{eV}$. The energy band gap of $3.24 \mathrm{eV}$ agrees to that reported in the literature for $\mathrm{ZnO}$ nanorods. Fluorescence spectroscopy confirmed the accuracy of the mathematical predictions since an emission at $380 \mathrm{~nm}(3.26 \mathrm{eV})$ was seen.

Keywords: ZnO nanorods, ZnO band gap, Kubelka-Munk method, rough surface

\section{Introduction}

Photo activated applications such as solar cells, $\mathrm{H}_{2}$ generators and photocatalysis, generally require the knowledge of the refractive index, absorption coefficient, band bap (BG) or any other optical parameters that serve to predict the performance of the employed materials ${ }^{1-5}$. The term BG is usually determined when the photo stimulated generation of electrons and holes at a certain wavelength is required, leading to a more accurate employment of the light sources and materials according to the selected application. In practice, the estimation of the BG becomes feasible within the use of indirect methods, commonly by the Tauc's plot, which demands the computation of the absorption coefficient $(\alpha)$. Then, $\alpha$ can be determined from the fitting or processing of ellipsometric or spectroscopic measurements ${ }^{1,2}$. According to literature, the employment of the ellipsometric technique is often recommended as it can lead to the determination of more reliable optical properties. However, it is still an indirect methodology that requests fitting models for the analysis of the data. Nevertheless, the available models are not suitable for all nanostructures, particularly when a rough surface has been developed ${ }^{1,2}$. In fact, this is why the utilization of the spectrophotometric technique combined with standard optical equations that require the measurement of only Transmittance and Reflectance is commonly accepted ${ }^{6-10}$. As a matter of fact, for those cases when a precise definition of the BG is desired or when possible substrate interference is detected, transport theories, specifically the KubelkaMunk (K-M) method, are employed ${ }^{3,4,11-13}$. Nevertheless, it

* e-mail: angelica.saenz@cimav.edu.mx is believed that such method has limited applicability and so, several modifications have resulted. Nonetheless, the adoption of alternative techniques implies a more complex examination of the data, comprising all possible surfaces and light interactions ${ }^{4,5,11}$. As a consequence and based on the simplicity of the spectrophotometric measurements, the adoption of the simple mathematical expressions remain a common practice in literature to report the BG value.

Therefore, since the variation in the BG is often claimed when the different optical equations or models are adopted, a comparative study among the various methods, including the K-M theory, was performed in order to estimate the BG value of a rough sample of $\mathrm{ZnO}$ nanorods and to establish applicability limitations of the formulas. $\mathrm{ZnO}$ in the form of nanorods was chosen because it is suitable in all of the aforementioned applications s, $5,7,9,10$ and its 1D morphology is preferably among other morphologies ${ }^{9,10}$.

\section{Materials and methods}

\subsection{Synthesis}

A sample consisting of $\mathrm{ZnO}$ nanorods was grown onto $\mathrm{TiO}_{2}$ coated borosilicate glass substrate (BGS), using the aerosol assisted CVD technique. The synthesis of the $\mathrm{ZnO}$ nanorods sample, Z, followed the conditions reported previously by Sáenz-Trevizo et al. ${ }^{15}$

\subsection{Characterization}

The crystalline phases were determined by grazing incidence x-ray diffraction (GIXRD). The experiment was 
carried out in a Panalytical X-Pert system, set at the following conditions: a $\mathrm{Cu} \mathrm{K} \alpha$ radiation $(\lambda=0.1542 \mathrm{~nm})$ operated at $40 \mathrm{keV}$ and $30 \mathrm{~mA}$, a grazing incidence angle of $0.5^{\circ}$, a scanning angle $2 \theta$ varied in range from $20^{\circ}$ to $80^{\circ}$, with a step size of $0.05^{\circ}$ and step time of $2.75 \mathrm{~s}$. Surface morphology was analyzed by scanning electron microscopy (SEM) in a field emission JEOL JSM-7401F microscope. The total, diffuse and collimated components of the reflectance and transmittance for the BGS substrate, $\mathrm{TiO}_{2}$ coated substrate and $\mathrm{ZnO}$ nanorods sample, were measured in the UV-VIS-NIR interval using a CARY 5000 spectrophotometer equipped with a DRA2500 integrating sphere accessory, as suggested in earlier studies ${ }^{11}$. Spectral collimated component was determined by subtracting the spectral diffuse component from the total measurement. Moreover, the fluorescence spectrum of the sample was measured using a modular spectrofluorometer Horiba Fluorolog-3, which uses a 450-W Xenon CW lamp as excitation source. The excitation wavelength was set at 325 $\mathrm{nm}$, while the interval of emission was in the range of 300 $\mathrm{nm}$ to $800 \mathrm{~nm}$ with a step of $5 \mathrm{~nm}$. The fluorescence signal was detected at a $45^{\circ}$ viewing mode for the incident light and that of emission. A slit of $1 \mathrm{~nm}$ was used for excitation and emission. The whole process was carried out at room temperature (293 K).

\section{Results and discussion}

\subsection{Crystalline structure, morphology and optical properties}

Diffractograph from the sample by GIXRD appears in Figure 1a. It is clear from the figure that sharp and defined peaks were detected during the analysis, which matched precisely with the synthesized tetragonal anatase and hexagonal wurtzite phases of $\mathrm{TiO}_{2}$ buffer thin film and $\mathrm{ZnO}$ layer of nanorods, respectively. Furthermore and as it can be seen in micrograph in Figure $1 \mathrm{~b}$, the as grown $\mathrm{ZnO}$ nanorods appeared mostly oriented following the [001] direction perpendicular to the substrate's surface, associated with the intense peak in Figure 1a that agrees with the (002) family planes of $\mathrm{ZnO}$. From Figure 1b, the columnar and hexagonal cross-section morphology of the $\mathrm{ZnO}$ nanorods was also evidenced. Results matched with the information presented earlier by Sáenz-Trevizo et al. ${ }^{15}$. Additionally, according to the morphology and orientation of the layer of nanorods, the development of a rough sample was considered.

Total transmittance $(\mathrm{T})$ and reflectance $(\mathrm{R})$ spectra of sample $\mathrm{Z}$ along with their diffuse components $\left(T_{D}\right.$ and $\left.R_{D}\right)$ appear in Figure 2. From this figure, it is noticeable that the main sample-light interactions are diffuse in both cases, associated with the roughness of the surface. Measured T and $\mathrm{R}$ were the properties of the stack $\mathrm{BGS}-\mathrm{TiO}_{2}-\mathrm{ZnO}$ nanorods layer considered for the analysis.
In order to evaluate the contribution of the bare BGS and $\mathrm{TiO}_{2}$ coated BGS on the optical properties of sample $\mathrm{Z}$, the total transmittance and reflectance spectra of both materials were measured. Results appear in Figure $3 \mathrm{a}$ and b, for bare BGS and $\mathrm{TiO}_{2}$ coated BGS, respectively. From the figure it is evident that for both cases, the collimated component of the reflectance and transmittance were mainly detected. This effect was related to the surface conditions, as its roughness was almost negligible even when a thin layer of $\mathrm{TiO}_{2}$ was grown onto the BGS. Moreover, it was also clear that the transmittance and reflectance profiles of $\mathrm{TiO}_{2}$ coated BGS, differ considerably from those of the bare BGS, corroborating the presence of the buffer thin film onto it. Noteworthy, the reflectance spectra of sample $\mathrm{Z}$ in Figure 2, presents a completely different profile to those seen on bare BGS and $\mathrm{TiO}_{2}$ coated BGS substrates in Figure 3, suggesting that the influence of the substrate is almost irrelevant when the optical properties of the stack structure are measured.

\subsection{Band Gap approximation using standard optical equations}

Several theoretical expressions named standard, as well as adaptations to the K-M method were used to compute $\alpha$ at each wavelength. All employed equations appear summarized in Table 1. Later on, $\alpha$ was used to estimate the optical BG energy using the Tauc's plot method ${ }^{16}$ following the relationship:

$$
\alpha E=B(E-E g)^{m}
$$

where $\mathrm{B}$ is a parameter independent of the photon energy $\mathrm{E}, \mathrm{Eg}$ is the optical band gap energy and the exponent $\mathrm{m}$ depends of the type of transition between bands. Since an allowed direct transition is considered for $\mathrm{ZnO}, m=1 / 2$ for our estimations.

Two common standard equations (M1 and M2) were used as initial approximations for BG estimation. Employed equations appear in Table 1. As all possible light - surface interactions are included in total $\mathrm{T}$ and $\mathrm{R}$ spectra, only these measured quantities were considered. Resulting Tauc's plots appear in Figure 4. For both intercepts over the energy axis of the extrapolated linear portion of the plots indicated a BG of $3.24 \pm 0.01 \mathrm{eV}$ for the nanostructure. Additionally, for M1 and M2 in Figure $4 \mathrm{a}$ and $\mathrm{b}$, no distinguishable differences were observed in the plotted profile, possibly due to the similarities of the employed formulas, including the natural logarithm, and even though the total reflectance was considered in M2. Slight variations can be observed for energies below the band gap energy, where the values of reflectance are higher. 

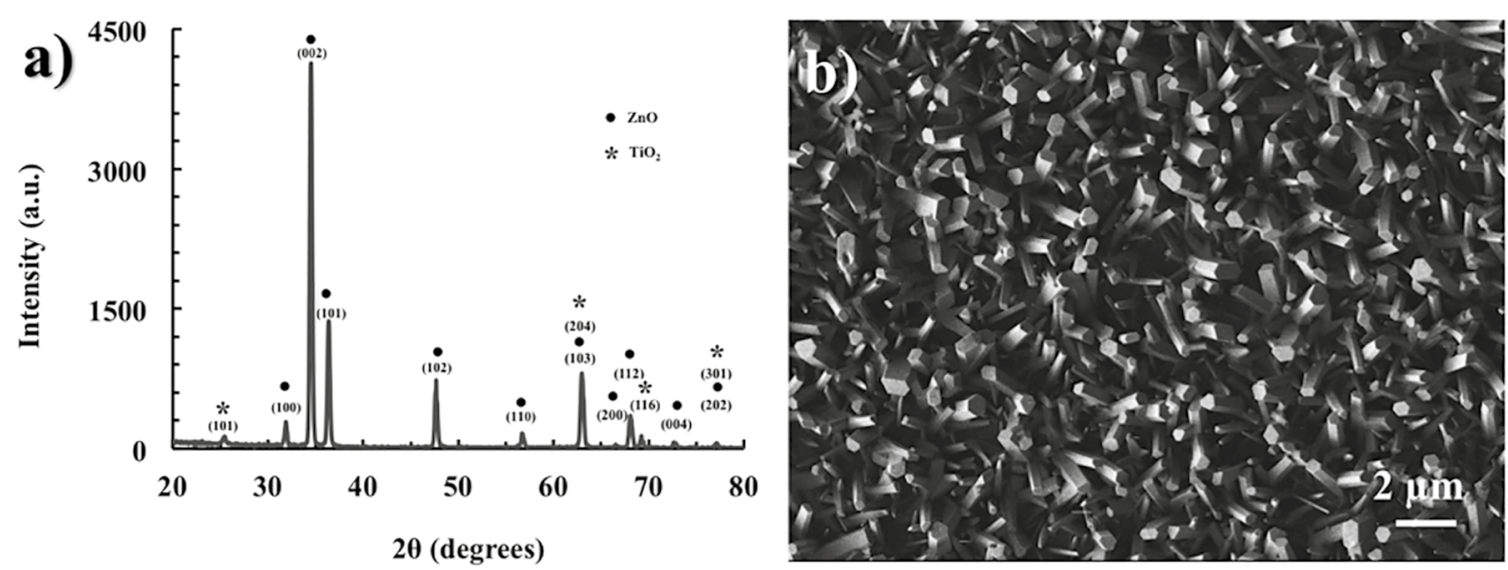

Figure 1. a) difractograph by GIXRD and b) SEM micrograph from sample Z. Both images reveal the crystalline structure of the synthesized materials and the topography of the surface.

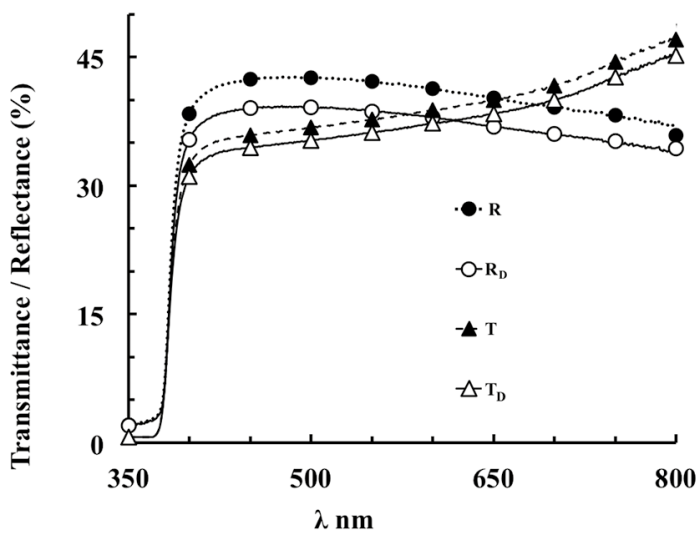

Figure 2. Total $(\mathrm{R}, \mathrm{T})$ and diffuse $\left(\mathrm{R}_{\mathrm{D}}, \mathrm{T}_{\mathrm{D}}\right)$ components of transmittance and reflectance spectra of sample $\mathrm{Z}$ in the UVVis region. Total components are mainly constituted by diffuse interactions in both cases.

\subsection{Band Gap determination using the Kubelka- Munk method}

Based on the development of a very rough surface, due to the existence of mainly diffuse interactions of the sample with light and despite the fact that incident light employed for the measurement of $\mathrm{T}$ and $\mathrm{R}^{11,12}$ was collimated, the application of the K-M method for BG estimation is suitable. Actually, two cases are applicable for the computation of $\alpha$ : the first one, neglecting the influence of the substrate (M3) and the second one, considering all substrate-coating interactions (M4). The application of M3 derived from the $\mathrm{K}-\mathrm{M}$ theory disregards any contribution from the substrate. This situation physically corresponds to a layer that is so thick that it is effectively opaque and so, hides the substrate completely ${ }^{12,13}$. The later assumption is supported with the
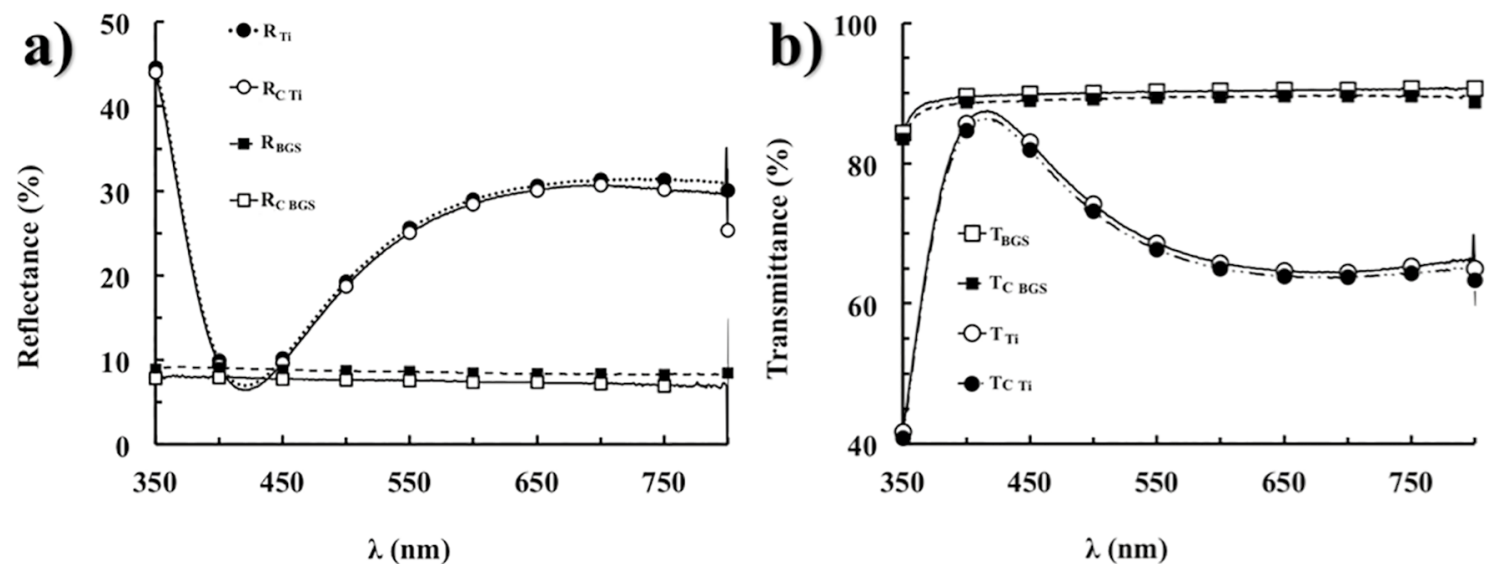

Figure 3. Optical properties of bare $\mathrm{BGS}$ and $\mathrm{TiO}_{2}$ covered $\mathrm{BGS}$ substrates. a) total $\left(\mathrm{R}_{\mathrm{BGS}}, \mathrm{R}_{\mathrm{Ti}}\right)$ and collimated $\left(\mathrm{R}_{\mathrm{CBGS}}, \mathrm{R}_{\mathrm{C} \text { Ti }}\right)$ components of reflectance spectra and $\mathrm{b}$ ) total $\left(\mathrm{T}_{\mathrm{BGS}}, \mathrm{T}_{\mathrm{Ti}}\right)$ and collimated $\left(\mathrm{T}_{\mathrm{C} \mathrm{BGS}}, \mathrm{T}_{\mathrm{C} \text { Ti }}\right)$ components of transmittance spectra. Total components are mainly constituted by specular interactions for both samples. 
Table 1. Formulas employed for the computation of the absorption coefficient according to several authors.

\begin{tabular}{|c|c|c|}
\hline Method & Equation & Special notes \\
\hline Method 1 (M1) & $\alpha=\frac{1}{t} \ln \left(\frac{1}{T}\right)$ & $\begin{array}{l}\text { Standard optical equation that considers the total } \\
\text { components of } T^{5,7,9} \text {. }\end{array}$ \\
\hline Method 2 (M2) & $\alpha=\frac{1}{t} \ln \left[\frac{(1-R)}{T}\right]$ & $\begin{array}{l}\text { Standard optical equation that considers the total } \\
\text { components of } T \text { and } R^{8} \text {. }\end{array}$ \\
\hline Method 3 (M3) & $\begin{array}{c}\alpha=\frac{1+R_{0}^{2}+T_{D}^{2}}{2 R_{0}} \\
R \infty=R_{0}+\frac{T_{D}^{2}}{2 b} \\
b=\sqrt{a^{2}-1} \\
S=\frac{1}{t\left(\frac{1}{R \infty}-R \infty\right)} \ln \left[\frac{\left(1-R \infty R_{0}\right) R \propto}{R \infty R_{0}}\right](\text { Equation } E-1) \\
S t=\frac{1}{b} \ln \frac{2 b R \infty}{T_{D}}(\text { Equation } E-2) \\
K=\frac{S(1-R \infty)^{2}}{2 R \infty}\end{array}$ & $\begin{array}{l}\text { K-M theory: case of an absorbing, intensely scattering } \\
\text { and thick material }{ }^{12-14} \text {. }\end{array}$ \\
\hline Method 4 (M4) & $\begin{array}{c}R_{K-M}=R_{0}+\frac{T_{D}^{2} R_{g}}{1-R_{g} R_{0}} \\
a=1 / 2\left(R+\frac{R_{0} R_{g}-R_{K-M}}{R_{0} R_{g}}\right) \\
S t=\frac{1}{b}\left(a \operatorname{coth} \frac{a-R_{K-M}}{b}-a \operatorname{coth} \frac{a-R_{g}}{b}\right) \\
K=S(a-1)\end{array}$ & $\begin{array}{l}\text { Formulas for practical use according to K-M theory } \\
\text { summarized by Kubelka }{ }^{13} \text {. }\end{array}$ \\
\hline
\end{tabular}

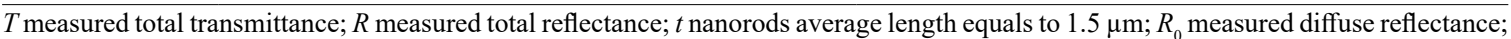
$T_{\mathrm{D}}$ measured diffuse transmittance; $R \infty$ reflectance of an infinite thick layer; $R_{K-M}$ total reflectance according to K-M equations; $S$ dispersion coefficient; $K$ absorption coefficient $=\alpha ; R g=R_{D B G S}$ measured reflectance of the substrate; $a$ and $b$ defined as K-M constants.
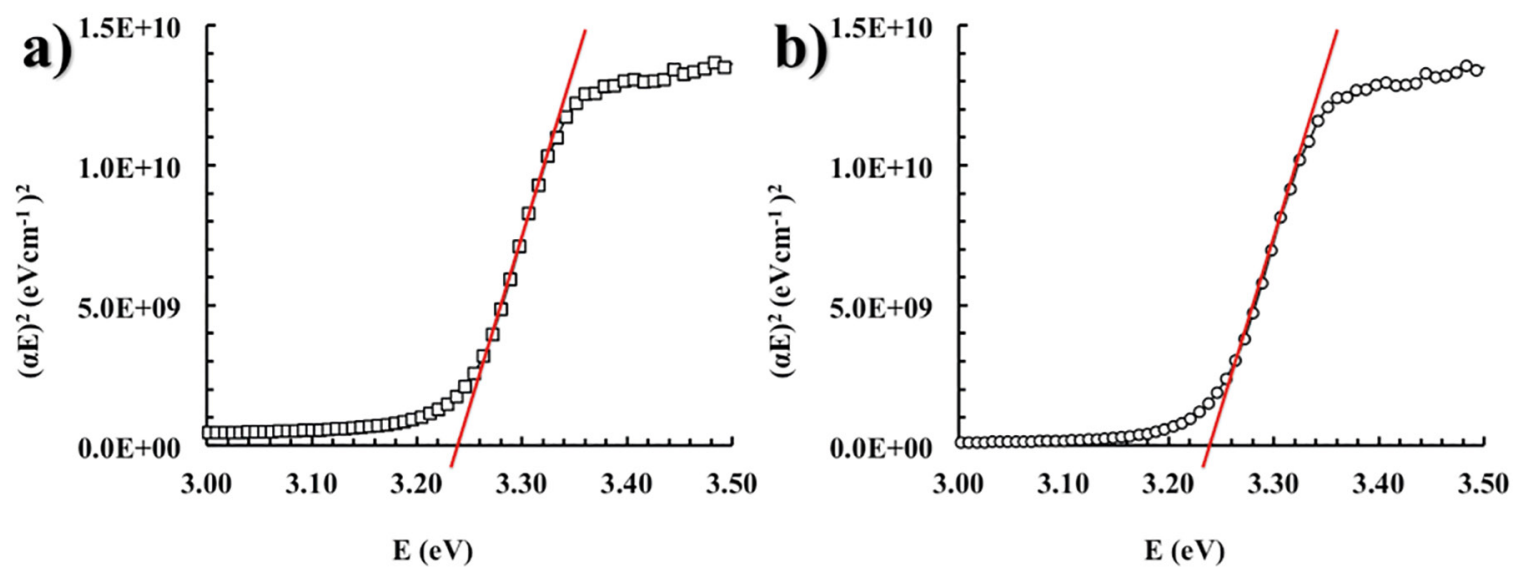

Figure 4. Tauc's plots obtained through the applications of standard equations in a) M1 and b) M2. All cases indicate a BG of $3.24 \pm 0.01 \mathrm{eV}$.

observations in Figures 2 and 3, from where it was seen that the main reflectance component of the sample was diffuse and also, that the measured transmittance and reflectance components of both possible substrates was collimated, exhibiting a different profile to that seen on the whole stack sample. Thus, these postulates simplify the mathematical expressions that are used to explain the optical properties of the studied layer of material, as agreed by several authors ${ }^{12-14}$.
Therefore, for the current case, only the measurement of $T_{D}$ and $R_{D}$, were needed. Noteworthy, the values of $T_{D}$ and $R_{D}$ closely resemble those values of $\mathrm{T}$ and $\mathrm{R}$ used in $\mathrm{M} 1$ and $\mathrm{M} 2$, since the diffuse components are predominant (see Figure 2). On the other hand, it was found in the literature that three possible routes for the computation of $S$ were available. From the three mathematical expressions, only one led to different values of S after its computation, exhibiting 
a deviation of about 11\% (Equations E-1 and E-2 in Table 1). However, both formulas had negligible impact on Tauc's plot as they generated the same BG values, around $3.24 \pm$ $0.01 \mathrm{eV}$. Results of the application of M3 appear in Figure 5.

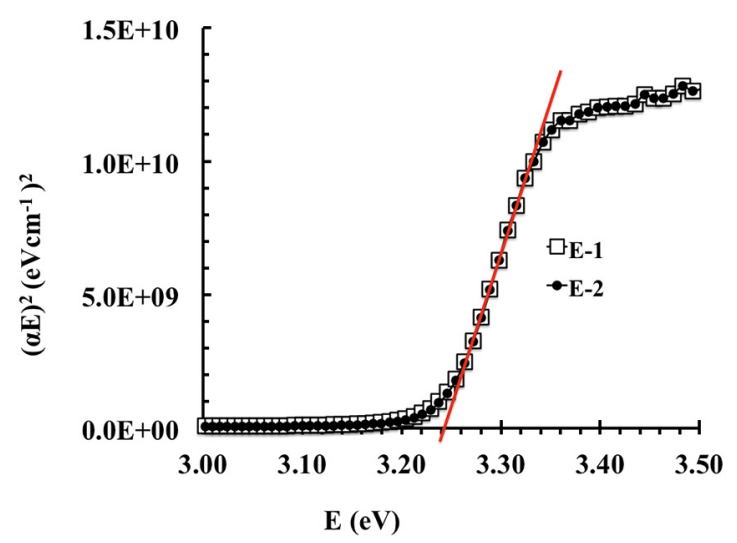

Figure 5. Tauc's plot obtained through the application of K-M case for an infinitely thick layer. The two mathematical expressions applied for estimation of S (E-1 and E-2) resulted in analogous plotted profiles.

An alternative route for BG estimation following K-M theory considers the contribution of the substrate (M4, results shown in Figure 6). For this case, a set of more complex equations become available and demand the knowledge of the substrate reflectance $\left(R_{\mathrm{g}}\right)$. As a first approximation we considered $R_{g}=\mathrm{R}_{\mathrm{DTi}}$, since the interaction of the substrate with the nanostructure or the air is different. Even though $R_{D T i}$ is almost insignificant (from Figure 3a), its inclusion resulted in an estimation of the optical BG of $3.17 \pm 0.01 \mathrm{eV}$. This value of BG implied a shift of the absorption to higher wavelengths (around $391 \mathrm{~nm}$ instead of $382 \mathrm{~nm}$ for $3.24 \mathrm{eV}$ ). Previous result suggests that $R_{g}$ should be lower than $\mathrm{R}_{\mathrm{DT}}$; , consistent with the fact that the refractive index of $\mathrm{ZnO}$ is higher than that of air, and closer to that of $\mathrm{TiO}_{2}$. Consequently, outcomes from methods M1-M4 are in good agreement with the optical BG determined for $\mathrm{ZnO}$ nanorods despite the substrate employed and the dimensions of the nanorods ${ }^{9,10}$.

The fluorescence spectrum of the stack sample was obtained in order to compare the energy required to stimulate fundamental electronic transitions on the $\mathrm{ZnO}$ nanorods, with the computed values of BG achieved within the application of all methods (M1-M4). Figure 7 shows the resulting spectrum, it is clearly seen that various emissions were detected. Emissions at $325 \mathrm{~nm}$ and $650 \mathrm{~nm}$ resulted from first and second order scattering due to the selected excitation wavelength, respectively. The observed emission at $380 \mathrm{~nm}$ (FWHM $20 \mathrm{~nm}$ ) was attributed to the direct recombination of electrons and holes within the material, suggesting a BG of approximately $3.26 \mathrm{eV}$, which matches at a great extent with the computed values resulting from M1 to M3. Additionally, a second order non linear emission related

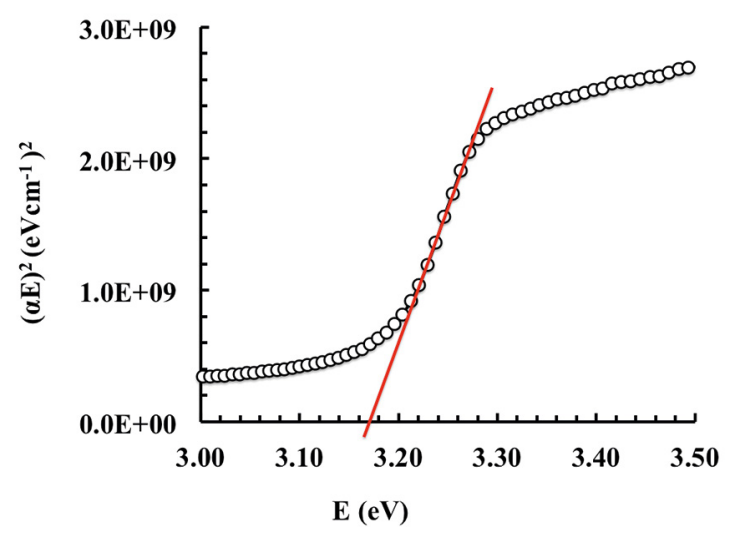

Figure 6. Tauc's plot obtained through the application of the formulas defined for general application of K-M method.

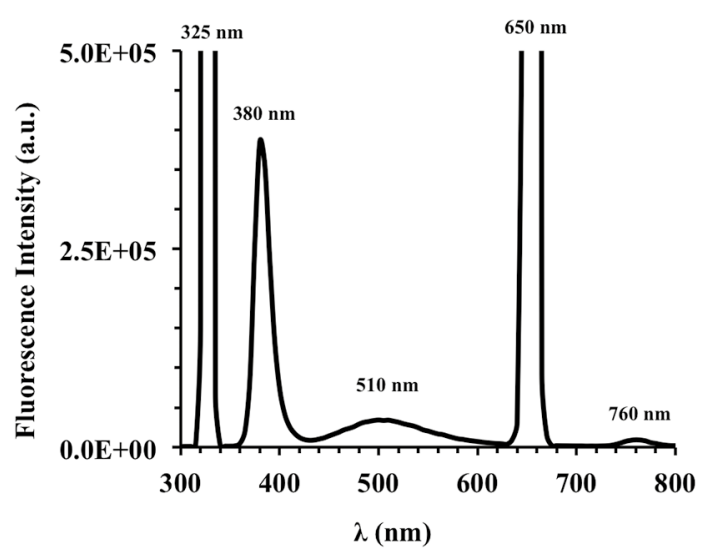

Figure 7. Fluorescence spectrum of sample $\mathrm{Z}$ obtained with excitation of $325 \mathrm{~nm}$. Emission at $380 \mathrm{~nm}$ closely resembles with the computed values of BG obtained with the application of methods M1, M2 and M3.

with previous phenomena was observed at $760 \mathrm{~nm}$. Finally, a broad peak centered at around $510 \mathrm{~nm}$ (FWHM $100 \mathrm{~nm})$ which could be associated to several mechanisms related with the structure of $\mathrm{ZnO}$, including transitions between singly ionized oxygen vacancies and photoexcited holes, surface defects, among others, as announced and previously observed in other reports ${ }^{17-19}$. Interestingly, these emissions in the visible region are not dependent on the morphology of the $\mathrm{ZnO}$. The fluorescent contribution of the substrate was not observed.

\section{Conclusions}

Despite total $T$ and $R$ from the stack sample consisted of mainly diffuse interactions, no differences among methods M1 through M3, for the derived values of the optical band gap, which was estimated as $3.24 \pm 0.01 \mathrm{eV}$. A narrower band gap of $3.17 \pm 0.01 \mathrm{eV}$ was estimated when incorporating 
the almost negligible $\mathrm{R}_{\mathrm{g}}$ as the substrate contribution. The computation of the BG by standard equations was corroborated by fluorescence spectroscopy, which indicated that approximately $3.26 \pm 0.09 \mathrm{eV}$ of energy was required for the excitation of $\mathrm{ZnO}$. Results indicated that all mathematical approximations are useful for the determination of the BG when other techniques are not available. However, it is appropriate to measure the optical properties of all involved surfaces in order to determine the nature of light-matter interactions.

\section{Acknowledgements}

The authors would like to thank SEP-CONACYT (project $\mathrm{N}^{\circ}$ 242612) for their partial financial support.

\section{References}

1. Selj JH, Mongstad T, Hauback BC, Karazhanov SZ. The dielectric functions and optical band gaps of thin films of amorphous and cubic crystalline $\mathrm{Mg}_{\sim 2} \mathrm{NiH}_{\sim 4}$. Thin Solid Films. 2012;520(22):6786-6792.

2. Hichou AE, Stein N, Boulanger C, Johann L. Structural and spectroscopic ellipsometry characterization for electrodeposited $\mathrm{ZnO}$ growth at different hydrogen peroxide concentration. Thin Solid Films. 2010;518(15):4150-4155.

3. Murphy AB. Optical properties of an optically rough coating from inversion of diffuse reflectance measurements. Applied Optics. 2007;46(16):3133-3143.

4. Curiel F, Vargas WE, Barrera RG. Visible spectral dependence of the scattering and absorption coefficients of pigmented coatings from inversion of diffuse reflectance spectra. Applied Optics. 2002;41(28):5969-5978.

5. Wang MD, Zhu DY, Liu Y, Lin Z, Zheng CX, He ZH, et al. Determination of Thickness and Optical Constants of ZnO Thin Films Prepared by Filtered Cathode Vacuum Arc Deposition. Chinese Physics Letters. 2008;25(2):743-746.

6. Vargas WE, Niklasson GA. Applicability conditions of the Kubelka-Munk theory. Applied Optics. 1997;36(22):5580-5586.

7. Sapkal RT, Shinde SS, Waghmode TR, Govindwar SP, Rajpure $\mathrm{KY}$, Bhosale $\mathrm{CH}$. Photo-corrosion inhibition and photoactivity enhancement with tailored zinc oxide thin films. Journal of Photochemistry and Photobiology B: Biology. 2012;110:15-21.
8. Tabbal M, Kim T, Woolf DN, Shin B, Aziz MJ. Fabrication and sub-band-gap absorption of single-crystal Si supersaturated with Se by pulsed laser mixing. Applied Physics A. 2010;98(3):589-594.

9. Foo KL, Hashim U, Muhammad K, Voon CH. Sol-gel synthesized zinc oxide nanorods and their structural and optical investigation for optoelectronic application. Nanoscale Research Letters. 2014;9(1):4291.

10. Krunks M, Katerski A, Dedova T, Acik IO, Mere A. Nanostructured solar cell based on spray pyrolysis deposited $\mathrm{ZnO}$ nanorod array. Solar Energy Materials and Solar Cells. 2008;92(9):1016-1019.

11. Murphy AB. Modified Kubelka-Munk model for calculation of the reflectance of coatings with optically-rough surfaces. Journal of Physics D: Applied Physics. 2006;39(16):3571-3581.

12. Džimbeg-Malčić V, Barbarić-Mikočević Ž, Itrić K. Kubelka-Munk theory in describing optical properties of paper (I). Tehnički Vjesnik/Technical Gazette. 2011:18(1):117-124.

13. Kubelka P. New contributions to the optics of intensely lightscattering materials. Part I. Journal of the Optical Society of America. 1948;38(5):448-457.

14. Lin H, Huang CP, Li W, Ni C, Shah SI, Tseng YH. Size dependency of nanocrystalline $\mathrm{TiO}_{2}$ on its optical property and photocatalytic reactivity exemplified by 2-chlorophenol. Applied Catalysis $B$ : Environmental. 2006;68(1-2):1-11.

15. Sáenz-Trevizo A, Amézaga-Madrid P, Fuentes-Cobas L, Pizá-Ruiz P, Antúnez-Flores W, Ornelas-Gutiérrez C, et al. Microstructural, chemical and textural characterization of $\mathrm{ZnO}$ nanorods synthesized by aerosol assisted chemical vapor deposition. Materials Characterization. 2014;98:215-221.

16. Stenzel O. The Physics of Thin Films Optical Spectra. Berlin: Springer-Verlag; 2005. 277 p.

17. Zhang Y, Mi L, Zheng Z. Study on the structure and optical property of $\mathrm{Zn}_{1-x} \mathrm{Cu} x \mathrm{O}$ sol-gel thin films on quartz substrate. Physica B: Condensed Matter. 2017;407(12):2254-2257.

18. Yue Q, Cheng J, Li G, Zhang K, Zhai Y, Wang L, et al. Fluorescence Property of $\mathrm{ZnO}$ Nanoparticles and the Interaction with Bromothymol Blue. Journal of Fluorescence. 2011;21(3):1131-1135.

19. Bekermann D, Ludwig A, Toader T, Maccato C, Barreca D, Gasparotto A, et al. MOCVD of ZnO Films from Bis(Ketoiminato) Zn(II) Precursors: Structure, Morphology and Optical Properties. Chemical Vapor Deposition. 2011;17(4-6):155-161. 